\title{
People's Perception of Domestic Service Robots: Same Household, Same Opinion?
}

\author{
Julia Fink, Valérie Bauwens, Omar Mubin, Frédéric Kaplan, and Pierre Dillenbourg \\ Ecole Polytechnique Fédérale de Lausanne, 1015 Lausanne, Switzerland \\ \{julia.fink, valerie.bauwens, omar.mubin, frederic.kaplan, \\ pierre.dillenbourg\}@epfl.ch
}

\begin{abstract}
The study presented in this paper examined people's perception of domestic service robots by means of an ethnographic study. We investigated initial reactions of nine households who lived with a Roomba vacuum cleaner robot over a two week period. To explore people's attitude and how it changed over time, we used a recurring questionnaire that was filled at three different times, integrated in 18 semi-structured qualitative interviews. Our findings suggest that being part of a specific household has an impact how each individual household member perceives the robot. We interpret that, even though individual experiences with the robot might differ from one other, a household shares a specific opinion about the robot. Moreover our findings also indicate that how people perceived Roomba did not change drastically over the two week period.
\end{abstract}

Keywords: attitudes towards robots, domestic service robots, human-robot interaction.

\section{Introduction}

Within the last years several service robots for personal and domestic use, such as vacuum cleaning robots, lawn mowing robots and toy robots have been introduced into the mass market. The number of domestic service robots deployed in homes increases constantly but lags behind early estimations. According to Bill Gates' article "A robot in every home", it seems that for the personal service robot industry, a similar scenario is going on as we had for the computer business 30 years ago [1]. On the one hand, there is a lack of common standards and platforms, so that robot developers usually have to start from scratch when building new robotic devices. On the other hand, when developing robots for domestic use, we cannot deny social implications of human-robot interaction, human behavior and people's expectations. It has been shown that social aspects play a crucial role in technology adoption [2] and that people tend to perceive artifacts showing intentional behavior as characters or even creatures [3]. The tendency to anthropomorphize nonhuman agents also holds for technical tools or robots that seem to lack capabilities of having social interaction [4]. An ethnographic study conducted by Forlizzi et al. revealed that a vacuum cleaning robot in contrast to a traditional vacuum cleaner deployed in the home changed people's cleaning activities and how they used other tools [5]. However, still little is known about how people actually use domestic service robots and how they react to 
them. The majority of literature on human-robot interaction in this area is of technical nature. But if we aim to develop and introduce into homes human-oriented robots that perform tasks for people in their everyday environment and safely co-exist with them, we need to understand not only technological affordances but also social aspects of human-robot interaction. In addition to more general issues about how people perceive domestic robots, questions arise about how people, children, the elderly and pets react to a robot in the home, what different kinds of difficulties they face, how they explore the robot and whether and how they integrate it into their daily life.

To address these questions, we gave a vacuum cleaning robot to nine households and followed people's experiences with the robot over two weeks. We decided to use iRobot's $\mathrm{sM}^{\mathrm{TM}}$ Roomba vacuum cleaning robot to study people's perception of it in homes because it has been available as a consumer product for several years. The robot is fully developed, quite robust and financially affordable. Surprisingly enough, even though, according to iRobot ${ }^{\mathrm{TM}}$, about 6 million units have been sold already, yet little is known about how people accept it. However, we argue that, acceptance of a domestic robot is not only related to the individual perspective but also to a certain 'household perspective' (e.g. a 'family perspective', such as shared values and beliefs). Thus we claim that, being member of a specific household (e.g. a family or a couple living together) could influence the acceptance and how people perceive the service robot in their home. To verify this, we analyzed people's attitudes and their reactions to the robot not only on an individual but also on a household level. A holistic view that regards people not only as users but rather as social actors within the ecology of their home will help to understand human-robot interaction in domestic settings $[5,6,7]$. We explored people's perceptions and examined how their reactions evolved. We visited each of the nine household twice, which made in total 18 semistructured interviews. We collected quantitative data in form of a questionnaire with rating scales filled out by all household members $(n=26)$ as well as qualitative data through interview conversations, home tours and on-site observations.

\section{$2 \quad$ Related Work}

In this part, we situate our project with respect to related work that studied the acceptance of technology and human-robot interaction in domestic spaces. Some studies have been carried out that focused on user needs for (future) domestic robots [8,9]. Participants of Scopelliti's study did not have a clear idea about what a future domestic robot could be or do in the household but rather responses seemed to emerge from science fiction movies or novels about robots [8]. The authors report some significant gender and age differences in the perception of domestic robots, for instance, in terms of confidence in the capabilities of robots, emotional reactions to a domestic robot, preferred characteristics and interaction modalities of robots. Based on the assessment of user needs, Sung et al. gave several suggestions in terms of design, namely that a domestic robot needs to provide a certain amount of human control, be compatible with the user's domestic environment, and take gender into consideration. In terms of social relation to the device, participants preferred a friendly designed robot that would act as a professional butler but not as a friend. Dautenhahn reported a similar finding and stated that people want to view their home robots as machines, assistants and servants, performing various tasks [10]. 
Several researchers studied social relationships to home technologies and domestic robots, such as intimacy, affective quality, and emotional attachment as well as their role during the process of adoption [11,2]. Social effects of technology adoption have been described as 'intimacy in computing' [3] or 'intimate ubiquitous computing' $[12,2]$ and despite using different technologies draw a similar conclusion: intimacy leads to greater acceptance of technology and perceived usability. It is likely that these effects also apply to robots such as Roomba $[6,10,13]$. It has been observed that domestic robots did not only lead people to change their cleaning patterns or the physical arrangement of their home but that they also developed relationships with the robot by assigning a name or ascribing personality traits to the device [6]. Intimacy can inform device adoption and help people to manage for example unreliability [13]. By feeling socially attached to the robotic vacuum cleaner, people were able to derive increased pleasure from cleaning, and expended effort to fit the robot into their homes. Scopelliti et al. concluded that acceptability of robotic devices in home settings, especially for elderly people, would not only depend on the practical benefits of the device, but on complex relationships between the cognitive, affective and emotional components of people's images of robots [8].

One concept often referred to when talking about the acceptance of robots is anthropomorphism. Epley et al. describe anthropomorphism as the tendency to "imbue the real or imagined behavior of nonhuman agents with humanlike characteristics, motivations, intentions or emotions" [4]. One of the derived determinants the authors name to explain when people are likely to anthropomorphize is people's desire for social contact and affiliation (sociality motivation). Anthropomorphism can thus play an important role concerning the acceptance of robots. It may serve as an effective method for improving usefulness and it has been shown that people were more likely to cooperate and work with a playful robot than with a serious robot [14].

Besides social relations between humans and robots, researchers also studied interactions between technological and social space in the home during the process of technology adoption: Venkatesh stressed the role of social space (e.g. family members, household activities, personal needs etc.) influencing the technological one [15]. He claimed that a thorough understanding of technology adoption in the household requires a theory of household behavior. These issues have more recently been studied by [16] for whom the composition of a household influences how people use a domestic service robot. The results of her survey study are based on the responses of more than 350 Roomba users.

In summary, the reviewed literature suggests that, for investigating service robots in domestic settings, it is worth considering not only users as individuals and looking for gender or age differences but also take into account the characteristics of the entire household. This hypothesis builds on the fact of a shared "physical" and "social space" in a domestic environment as well as on findings from previous studies showing that the household composition affected the expectation of Roomba as a practical tool [7]. Further, we assume that the adoption of a domestic robot in the context of the home not only happens as an individual but as a whole household [11]. In our study, we therefore apply a holistic household view and quantitatively argue for doing so. 


\section{Study Design}

\subsection{Method and Measurements}

Our study aimed at exploring people's perception of a domestic robot they have in their home for two weeks. We combined quantitative and qualitative research methods which enabled us to deeply explore participant's beliefs and reactions to robots. A part of the methodology of our approach was similar to that followed by Sung et al. and we adapted a questionnaire from her research [17].

Following a user-centered approach, we implemented a recurring questionnaire into several semi-structured interviews with the participants. The interviews took place at each participant's home and lasted about 1.5 hours each. They were audio recorded and qualitatively re-transcribed. We asked all household members to be present during our household visits; however, this was not always the case. To enhance discussion and examine social roles in the household, participants of the same household were interviewed collectively, thus children were not separated from their parents, for instance. In total, each of the nine households was visited twice during the two weeks which makes in total 18 visits. In addition to that, we have conducted a preliminary interview one week prior to the study, including a home tour. We got to know the household, the people and pets living in the household, asked them about their attitude towards technology and robots as well as their cleaning routine and the structure of their daily life. For the following two visits we took the questionnaire with us each time and asked participants to rank Roomba on a seven point Likert scale (1-7) in respect to its intelligence, usefulness, ease of use, experienced fun, expected impact on the household and the participant's overall impression. A questionnaire with Likert rating scales to assess people's attitude towards robots has also been used by [8, 17].

Participants filled out the questionnaire three times:

- Before they had seen Roomba (T1), in order to investigate their expectations and imaginations of the vacuuming cleaning robot;

- After they had unpacked and interacted with Roomba for about 10 to 15 minutes (T2) to catch their initial impression;

- Two weeks after they received Roomba (T3).

The participants' ratings on the questionnaires were filled in a spreadsheet, regarding one decimal steps where two coders agreed on the exact interpretation of each mark (e.g. if the mark was understood as 5.4 or 5.5).

\subsection{Participants}

Our sample consisted of nine households with a total of 30 participants. Four of the fifteen children were six years and younger, so we did not ask them to evaluate the robot using the questionnaire. 26 participants filled out the questionnaire: 14 men and 12 women, thereof 11 children, and 15 adults, ranging from 7 to 70 years old. The mean age of all participants was 30, of all adults older than 18 years the mean age was 43. More specifically, we had three single-headed (one single parent) and six doubleheaded households. Six households had children from six months to 18 years old. 
Finally, since our study focused on cleaning, we recruited households with pets ( $\mathrm{n}=5$ ), such as dogs and cats, and households with cleaning services $(n=4)$. Among the four who hired cleaning services, three received the service once a week and one every other week. Everyone of the quite heterogeneous sample lived in the area of Lausanne in the French speaking part of Switzerland. With more than one third of its inhabitants being foreigners, Lausanne displays multi-faceted cultures besides a Swiss-French mentality. Our sample reflects this cultural diversity including people of American, Austrian, British, Danish, French, German, and Swedish background besides two Swiss households. Interviews and questionnaires were carried out in English, French or German, depending on the language all household members spoke fluently.

\subsection{Design}

Our previously mentioned questionnaire has been used to evaluate the perception of Roomba by the households. The quantitative analysis was divided in three parts. The first analysis was run as a simple repeated measures ANOVA with time as the independent variable. The second analysis included a number of between subject factors that were also incorporated in a mixed design ANOVA. For the first two parts of the analysis we considered every individual household member as an individual data point. In the third part of our analysis we aggregated all the members of one household to make a single data point and a repeated measure ANOVA was done. In the third part the single data point would represent the household opinion (which for the case of our two single households displayed the opinion of a single person).

\section{$4 \quad$ Findings}

Our overall aim was to find out how people and households perceive a domestic robot in their home. This section is structured in two parts. First, we present the overall results of how our participants on an individual level perceived the robot in respect to its intelligence, usefulness, usability, fun, attachment, impact, and their overall impression. In the second part we present our findings on a household level aggregating data of individual members to verify our assumption that response behavior is influenced by the fact of being part of a specific household. Although in general how people rated Roomba in terms of the provided topics (Table 1) didn't change significantly over time, how they qualitatively described the robot changed with time. These qualitative results will be reported elsewhere.

\subsection{Individual Level}

We first present the quantitative analysis on an individual level over time. Table 1 shows the means and standard deviations of each topic over the three time points. Note that for some individuals we did not have ratings for all three occasions. Therefore they had to be dropped from the ANOVA analysis. Thus, our sample size was 19. 
Table 1. Means and standard deviations for each topic over the three times. Repeated measures ANOVA with time as within subjects factor.

\begin{tabular}{|c|c|c|c|c|c|c|c|c|}
\hline Topic & $\begin{array}{l}\mathrm{T}_{1} \\
\text { Mean }\end{array}$ & $\begin{array}{l}\mathrm{T}_{1} \\
\text { Std dev }\end{array}$ & $\begin{array}{l}\mathrm{T}_{2} \\
\text { Mean }\end{array}$ & $\begin{array}{l}\mathrm{T}_{2} \\
\text { Std dev }\end{array}$ & $\begin{array}{l}\mathrm{T}_{3} \\
\text { Mean }\end{array}$ & $\begin{array}{l}\mathrm{T}_{3} \\
\text { Std dev }\end{array}$ & $\begin{array}{l}F \\
(2,36)\end{array}$ & $\mathrm{p}$ \\
\hline Intelligence & 4.38 & 1.68 & 4.63 & 1.7 & 4.22 & 1.71 & 0.70 & 0.50 \\
\hline Usefulness & 5.2 & 1.39 & 5.44 & 1.09 & 5.0 & 1.36 & 0.81 & 0.45 \\
\hline Ease of use & 5.74 & 1.09 & 6.28 & 0.63 & 6.02 & 1.25 & 1.59 & 0.22 \\
\hline Fun & 4.38 & 1.70 & 4.43 & 1.7 & 4.43 & 1.72 & 0.01 & 1.0 \\
\hline Attachment & 3.8 & 1.43 & 4.28 & 1.88 & 4.23 & 1.71 & 0.92 & 0.41 \\
\hline Impact & 4.26 & 1.74 & 4.56 & 1.59 & 4.17 & 1.29 & 0.62 & 0.55 \\
\hline $\begin{array}{l}\text { Overall } \\
\text { impression }\end{array}$ & 5.43 & 1.21 & 5.56 & 1.21 & 4.95 & 1.29 & 1.28 & 0.29 \\
\hline
\end{tabular}

We executed a repeated measures ANOVA with time as the within subjects factor. As evident by Table 1, time did not have a significant effect on any of the topics. There is however an interesting pattern that for all of the seven topics, the T2 mean value is the maximum value.

After testing the main effects we aimed to determine the impact of any external factors or biases on an individual's rating. The factors were considered one at a time because if we had included them together in a mixed design ANOVA this would have reduced the sample size even further for every combination.

We first wished to examine any gender effects. Surprisingly, gender did not have a significant effect on the ratings (see Table 2). We would have expected male participants giving more positive ratings than female participants purely based on the fact that Roomba is a technological device. However, in our qualitative observations it turned out that female householders were the primary users of Roomba and this fact could have resulted in females giving equally high ratings as compared to men. This goes along with results of $[10,16]$. However, other studies reported significant gender differences [8]. We further analyzed variances for different age groups and pet owners but could not find any significant results. This might be due to the rather small size of the sample within each age group.

Table 2. Mixed design ANOVA with time as within subjects factor and gender as between subjects factor. ( $\mathrm{f}=$ female; $\mathrm{m}=$ male)

\begin{tabular}{lllllllll}
\hline Topic & $\begin{array}{l}\mathrm{T}_{1} \mathrm{~m} \\
\text { Mean }\end{array}$ & $\begin{array}{l}\mathrm{T}_{1} \mathrm{f} \\
\text { Mean }\end{array}$ & $\begin{array}{l}\mathrm{T}_{2} \mathrm{~m} \\
\text { Mean }\end{array}$ & $\begin{array}{l}\mathrm{T}_{2} \mathrm{f} \\
\text { Mean }\end{array}$ & $\begin{array}{l}\mathrm{T}_{3} \mathrm{~m} \\
\text { Mean }\end{array}$ & $\begin{array}{l}\mathrm{T}_{3} \mathrm{f} \\
\text { Mean }\end{array}$ & $\begin{array}{l}\mathrm{F} \\
(1,17)\end{array}$ & $\mathrm{p}$ \\
\hline Intelligence & 5.07 & 3.62 & 5.02 & 4.19 & 4.68 & 3.72 & 2.82 & 0.11 \\
Usefulness & 5.81 & 4.52 & 5.84 & 4.99 & 4.95 & 5.00 & 3.13 & 0.10 \\
Ease of use & 6.26 & 5.17 & 6.39 & 6.17 & 5.93 & 6.11 & 1.52 & 0.24 \\
Fun & 5.05 & 3.63 & 4.53 & 4.31 & 4.64 & 4.19 & 1.24 & 0.28 \\
Attachment & 3.49 & 3.92 & 4.14 & 4.21 & 3.81 & 4.69 & 0.58 & 0.46 \\
Impact & 3.67 & 4.26 & 4.59 & 4.39 & 4.34 & 3.99 & 0.00 & 0.98 \\
$\begin{array}{l}\text { Overall } \\
\text { impression }\end{array}$ & 5.75 & 4.86 & 6.00 & 5.10 & 4.82 & 5.10 & 2.89 & 0.11 \\
\hline
\end{tabular}




\subsection{Household Level}

The next factor was family code which indicated to which particular household an individual belongs. We executed a mixed design ANOVA with time as the within subject factor and family code as the between subject factor. The results are summarized in Table 3.

Table 3. ANOVA results showing the effect of family code on the ratings

\begin{tabular}{lll}
\hline Topic & $\begin{array}{l}\mathrm{F} \\
(1,10)\end{array}$ & $\mathrm{p}$ \\
\hline Intelligence & 2.68 & 0.07 \\
Usefulness & 3.9 & $0.02^{*}$ \\
Ease of use & 1.89 & 0.16 \\
Fun & 9.77 & $0.02^{*}$ \\
Attachment & 9.83 & $0.02^{*}$ \\
Impact & 6.45 & 0.16 \\
Overall & 1.88 & 0.27 \\
\hline
\end{tabular}

The results of the ANOVA showed that for some of the topics, being part of a particular household had a significant effect. As an example for this, Figure 1 shows individual responses for each member of household F9. Consisting of four household members (single father and three boys from eight to eleven years old) the graph illustrates how the individual ratings form a certain family opinion, where in this case, one family member (P19) differed in his responses from the others. For this family, there was clearly no peak at T2.

F9 Usefulness

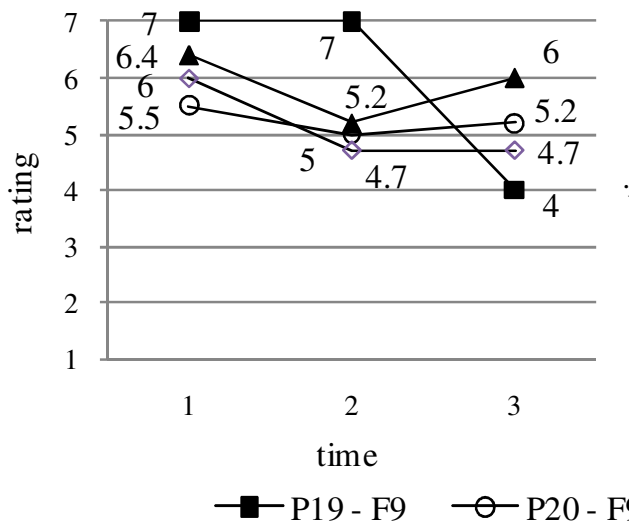

F9 Fun

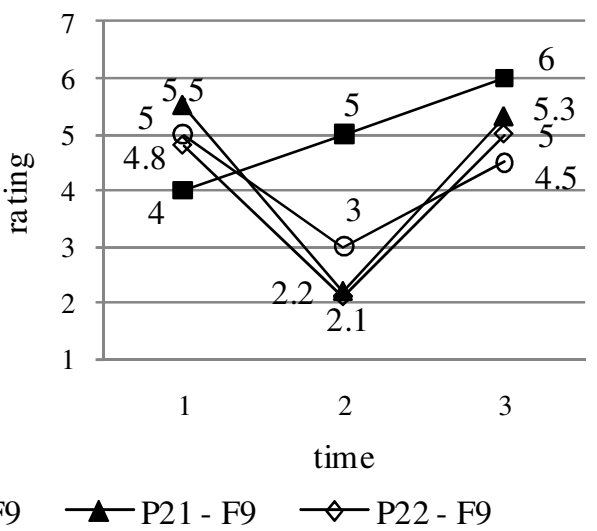

Fig. 1. Individual responses for usefulness and fun for each of the four members of a family

We wanted to examine in more detail how belonging to a family influences people's response behavior and carried out further analyses by aggregating the scores 
of the individual members of a single family. We carried out a repeated measures ANOVA on the aggregated scores of the family with time as the within subject (family) factor. However, we did not get any significant results for this case.

In conclusion, our data shows that during two weeks, on an individual level, there have not been any significant changes in how people rated their domestic robots in respect to intelligence, usefulness, ease of use, fun, attachment, impact and the overall impression. However, the rating of various topics was significantly influenced by the fact that participants belonged to the same household. Thus, on a household level, we can report significant effects on people's perception of robots. Another interesting pattern that emerged was that for individuals we could see a peak effect. The mean rating for time $\mathrm{T} 2$ was higher than $\mathrm{T} 1$, but $\mathrm{T} 3$ was always less than or equal to $\mathrm{T} 2$.

\section{$5 \quad$ Limitations and Discussion}

Our aim was to study human-robot interaction including social aspects in a setting that was as natural as possible. Conducting qualitative research in a domestic site raises various challenges, such as to capture reliable data in a highly uncontrolled environment with constraints of privacy and temporality in the home. Furthermore, in our study responses might be biased due to the fact that people received a cleaning robot from us and that they did not have to make a financial investment with buying the device. This might have influenced how people perceived the robot.

The interpretability of our results is limited by the sample size counting nine households with 30 participants but several missing responses. However, a study on a larger scale would not have been realizable due to time and resource constraints. We nevertheless analyzed data on a household level and hypothesized that being part of a particular household influences people's perception of robots. It remains to mention that two of the nine households consisted of one person only. In addition to the heterogeneity of participants might have contributed to the observed effect. Cultural differences in the perception of robots have been investigated by [22].

As mentioned before, this study is part of a longitudinal ethnography. It documents the evolution of people's perception of robots for only two weeks. The responses are likely to portray certain novelty effects which can be described as the first responses to a technology $[17,19]$. Covering a period of two weeks, data does not allow drawing conclusions in terms of usage patterns or continuing adoption of the device. Longterm usage of robots and usage patterns beyond novelty effects have been examined by $[7,17,19,20]$. We didn't find a significant change in how people overall rated the robot during the two weeks and assume that amongst others, novelty factors might be one reason for this. Literature suggests that people's expectations are quite powerful in shaping the initial experience [6] and this in turn seems to be crucial for the further process of adoption [11]. Another reason why the perception did not change drastically over time might be that Roomba could have been perceived more as a technical device rather than as a social robot. Roomba lacks the ability to learn and monotonously performs the task of vacuuming. It remains to see how far people's rating of Roomba changes over a longer period of time or whether with Roomba people's perception remains quite stable after people have formed first opinions. 
We presented findings about how people perceive a domestic robot on an individual level as well as on a household level and hypothesized different effects.

- On an individual level we did not find significant gender or age effects. This was surprising but might be explained through results on the household level.

- On a household level we found a significant effect that we called 'family effect' and speculate about their characteristics in terms of the shared social values (H1), the shared physical environment (H2) or an opinion leader effect (H3).

The observed 'family effect' can be impacted by the fact that the members of one household have not been separated during the interviews. More important, the effect may result from social norms, values and beliefs that are shared in one household (H1) but also be due to the physical layout of the home (H2). The fact that an apartment has stairs or an open kitchen may have an effect on the relevance of Roomba, for instance. Similarly, Severinson-Eklundh argues that understanding human-robot relationship includes consideration of the group of people involved, their social norms, roles and beliefs, as well as the shared physical environment [18]. Besides this, we saw that, while families rated Roomba, there tended to be an opinion leader (H3) who influenced the others. In social science, group conformity and decision making has been extensively studied since the 1950ies (e.g. Asch's experiment on group conformity). It will be important in further studies to try to disentangle the various effects we encounter in domestic environments.

Acknowledgements. We thank all our participants for their time and engagement in this study. We also thank iRobot ${ }^{\mathrm{TM}}$ and iRobotics $\mathrm{GmbH}$ for their support. Further, we thank JaYoung Sung and researchers from Georgia Tech for sharing their experience with us. This research was supported by the Swiss National Science Foundation through the National Centre of Competence in Research on Robotics.

\section{References}

1. Gates, B.: A Robot in Every Home. In: Scientific American, pp. 58-65 (January 2007)

2. Venkatesh, V.: Determinants of Perceived Ease of Use: Integrating Control, Intrinsic Motivation, and Emotion into the Technology Acceptance Model. Information Systems Research 11(4), 342-365 (2000)

3. Reeves, B., Nass, C.: The Media Equation: How People Treat Computers, Television, and New Media like Real People and Places. Cambridge University Press, New York (1996)

4. Epley, N., Waytz, A., Cacioppo, J.T.: On seeing human: A three-factor theory of anthropomorphism. Psychological Review 114(4), 864-886 (2007)

5. Forlizzi, J.: How Robotic Products Become Social Products: An Ethnographic Study of Cleaning in the Home. In: HRI 2007 Proceedings, Arlington, VA, USA, pp. 129-136. ACM Press (2007)

6. Forlizzi, J., DiSalvo, C.: Service Robots in the Domestic Environment: A Study of the Roomba Vacuum in the Home. In: HRI 2006 Proceedings, Salt Lake City, UT, USA, pp. 258-265. ACM Press (2006)

7. Sung, J., Grinter, R.E., Christensen, H.I.: Domestic Robot Ecology. International Journal of Social Robotics 2(4), 417-429 (2010) 
8. Scopelliti, M., Giuliani, M.V., Fornara, F.: Robots in a domestic setting: a psychological approach. Journal Universal Access in the Information Society 4(2), 146-155 (2005)

9. Sung, J., Christensen, H.I., Grinter, R.E.: Sketching the Future: Assessing User Needs for Domestic Robots. In: IEEE International Symposium on Robot and Human Interactive Communication, Toyama, Japan, pp. 153-158 (2009)

10. Dautenhahn, K., Woods, S., Kaouir, C., Walters, M.L., Koay, K.L., Werry, I.: What is a Robot Companion - Friend, Assistant or Butler? In: IEEE Robotics Society of Japan International Conference on Intelligent Robots and Systems (2005)

11. Rogers, E.M.: Diffusion of Innovations. Fifth edn. Free Press, New York (2003)

12. Bell, G., Blythe, M., Gaver, B., Sengers, P., Wright, P.: Designing Culturally Situated Technologies for the Home. In: CHI 2003 Proceedings, Ft. Lauderdale, FL, USA, pp. 1062-1063. ACM Press (2003)

13. Sung, J., Guo, L., Grinter, R.E., Christensen, H.I.: "My Roomba Is Rambo": Intimate Home Appliances. In: Krumm, J., Abowd, G.D., Seneviratne, A., Strang, T. (eds.) UbiComp 2007. LNCS, vol. 4717, pp. 145-162. Springer, Heidelberg (2007)

14. Kiesler, S., Goetz, J.: Mental models of robotic assistants. In: CHI 2002 Proceedings, Minneapolis, MN, USA, pp. 576-577. ACM Press (2002)

15. Venkatesh, A.: Computers and Other Interactive Technologies for the Home. Communications of the ACM 39(12), 47-54 (1996)

16. Sung, J., Grinter, R.E., Christensen, H.I., Guo, L.: Housewives or Technophiles?: Understanding Domestic Robot Owners. In: HRI 2008 Proceedings, Amsterdam, The Netherlands, pp. 129-136. ACM Press (2008)

17. Sung, J., Christensen, H.I., Grinter, R.E.: Robots in the Wild: Understanding Long-term Use. In: HRI 2009 Proceedings, La Jolla, CA, USA, pp. 45-52. ACM Press (2009)

18. Severinson-Eklundh, K., Green, A., Hüttenrauch, H.: Social and collaborative aspects of interaction with a service robot. Robotics and Autonomous Systems 42(3-4), 223-234 (2003)

19. Hüttenrauch, H., Severinson-Eklundh, K.: Fetch-and-carry with CERO: Observations from a long-term study with a service robot. In: IEEE International Workshop on Robot and Human Interactive Communication, Berlin, Germany, pp. 158-163 (2002)

20. Koay, K.L., Syrdal, D.S., Walters, M.L., Dautenhahn, K.: Living with Robots: Investigating the Habituation Effect in Participants' Preferences During a Longitudinal HumanRobot Interaction Study. In: IEEE International Conference on Robot and Human Interactive Communication, Jeju, Korea, pp. 564-569 (2007)

21. Venkatesh, A., Kruse, E., Chuan-Fong Shih, E.: The networked home: an analysis of current developments and future trends. Cognition, Technology, and Work 5, 23-32 (2003)

22. Kaplan, F.: Who is afraid of the humanoid? Investigating cultural differences in the acceptance of robots. International Journal of Humanoid Robotics 1(3), 1-16 (2004) 